\title{
Complete Genome Resources for Xylella fastidiosa Strains AlmaEM3 and BB08-1 Reveal Prophage-Associated Structural Variation Among Blueberry-Infecting Strains
}

\author{
Michael L. O'Leary, ${ }^{1, \dagger}$ Luis F. Arias-Giraldo, ${ }^{2}$ Lindsey P. Burbank, ${ }^{1}$ Leonardo De La Fuente ${ }^{3}$ \\ and Blanca B. Landa ${ }^{2}$ \\ ${ }^{1}$ United States Department of Agriculture-Agricultural Research Service, San Joaquin Valley Agricultural \\ Sciences Center, Parlier, CA 93648, U.S.A. \\ ${ }^{2}$ Institute for Sustainable Agriculture, Consejo Superior de Investigaciones Científicas (IAS-CSIC), \\ Córdoba, Spain \\ ${ }^{3}$ Department of Entomology and Plant Pathology, Auburn University, Auburn, AL 36849, U.S.A.
}

\section{Resource Announcement}

Xylella fastidiosa is a Gram-negative, xylem-limited, plant-pathogenic bacterium that causes disease on a diverse set of agriculturally important hosts. $X$. fastidiosa strains can be divided into subspecies and sequence types (STs) by genetic analysis and, although $X$. fastidiosa as a species has a wide host range, the host on which any one genotype causes disease appears to be limited. In the southeastern United States (i.e., Alabama, Florida, Georgia, and Louisiana), $X$. fastidiosa is the causal agent of bacterial leaf scorch disease on southern highbush blueberry, inducing marginal leaf chlorosis, necrosis, defoliation, and a "yellow-twig" phenotype on some cultivars, resulting in significant yield losses (Chang et al. 2009; Ferguson et al. 2017; Harmon and Hopkins 2009). Genetic characterization of $X$. fastidiosa strains infecting blueberry has revealed that, although the majority of strains associated with bacterial leaf scorch disease belong to $X$. fastidiosa subsp. multiplex, strains belonging to $X$. fastidiosa subsp. fastidiosa can cause bacterial leaf scorch of blueberry in both greenhouse (Burbank et al. 2019; Oliver et al. 2015) and field (Di Genova et al. 2020) conditions.

Relatively few $X$. fastidiosa subsp. multiplex strains that cause bacterial leaf scorch of blueberry have genome sequences available. These strains include AlmaEM3, an ST 42 strain isolated from resistant blueberry cultivar 'Emerald' grown in Georgia, United States in 2011; and BB08-1, an ST 43 strain isolated from the susceptible cultivar 'Windsor' grown in Florida, United States in 2008 (Oliver et al. 2014; Vanhove et al. 2019). The BB08-1 draft assembly present in GenBank (accession GCA_006369965.1) contains many frameshifted coding sequences which, in context of the sequencing platform used and low sequencing depth for this strain, are likely sequencing artifacts (Potnis et al. 2019). Analysis with CheckM identified multiple copies of a small number of marker genes (Table 1), raising the possibility that this assembly may have derived from a strain admixture (Parks et al. 2015); alternatively, this may be related to assembly quality. Thus, to facilitate future comparisons between blueberry-infecting $X$. fastidiosa strains, we used a hybrid sequencing approach to generate high-quality complete sequences for AlmaEM3 and BB08-1.

\section{${ }^{\dagger}$ Corresponding author: M. L. O’Leary; michael.oleary@usda.gov}

Mention of trade names or commercial products in this publication is solely for the purpose of providing specific information and does not imply recommendation or endorsement by the U.S. Department of Agriculture. USDA is an equal opportunity provider and employer.

The author(s) declare no conflict of interest.

Accepted for publication 22 August 2021.

\section{Funding}

This work was funded by the United States Department of Agriculture-Agricultural Research Service appropriated project 2034-22000-012-00D and the Xylella fastidiosa Active Containment Through a Multidisciplinary-Oriented Research Strategy (XF-ACTORS) project. Illumina sequencing for BB08-1 was carried out at the University of California, Davis, Genome Center DNA Technologies and Expression Analysis Cores, supported by National Institute of Health Shared Instrumentation Grant 1S100D010786-01.

\section{Keywords}

bacterial pathogens, bioinformatics, genomics, microbe-genome sequencing 
Table 1. Assembly features and comparison with draft assemblies

\begin{tabular}{|c|c|c|c|c|}
\hline \multirow[b]{3}{*}{ Features } & \multicolumn{4}{|c|}{ Assembly source per strain } \\
\hline & \multicolumn{2}{|c|}{ AlmaEM3 } & \multicolumn{2}{|c|}{ BB08-1 } \\
\hline & This study & Potnis et al. 2019 & This study & Potnis et al. 2019 \\
\hline Contigs & 1 & 30 & 1 & 98 \\
\hline Total sequencing depth & $192 x$ & $50 x$ & $1,370 x$ & $17 x$ \\
\hline GC content (\%) & 51.80 & 51.70 & 51.80 & 51.70 \\
\hline Transfer RNAs & 49 & 50 & 49 & 63 \\
\hline Complete ribosomal RNA operons & 2 & 2 & 2 & 4 \\
\hline IDEEL $<0.9^{\mathrm{C}}$ & 170 & 165 & 194 & 543 \\
\hline \multicolumn{5}{|l|}{ CheckM } \\
\hline Completeness (\%) & 99.64 & 99.64 & 99.60 & 99.53 \\
\hline Contamination (\%) & 0 & 0 & 0 & 5.11 \\
\hline
\end{tabular}

\footnotetext{
${ }^{a} \mathrm{ANI}=$ average nucleic acid identity and ${ }^{\top}$ denotes type strain.

${ }^{\mathrm{b}}$ Coding sequence (CDS) annotated with NCBI Prokaryotic Genome Annotation Pipeline.

${ }^{\mathrm{c}}$ Length ratio $<0.9$ suggests that a protein-coding sequencing may be erroneously disrupted (i.e., an uncorrected frameshift).
}

For AlmaEM3, DNA was extracted using the Qiagen DNeasy Plant Mini kit whereas, for BB08-1, DNA was extracted using a Qiagen DNeasy Blood and Tissue kit, including a treatment with the addition of $4 \mu \mathrm{l}$ of RNaseA (100 $\mathrm{mg} / \mathrm{ml}$ ). DNA was quantified via Qubit and, prior to library preparation, DNA for BB08-1 was sheared to approximately 8-kb fragments with a Covaris $\mathrm{g}$-tube. Sequencing libraries for both strains were prepared using a ligation sequencing kit (LSK-109; Oxford Nanopore). Libraries were sequenced in multiplexed runs on MinION devices using R9.4.1 flow cells. Sequencing reads for AlmaEM3 and BB08-1 were basecalled with Guppy v3.2.6 with default parameters or v3.0.3 with parameters -qscore_filtering -min_qscore 7, respectively, and trimmed with Porechop v0.2.4 (Wick et al. 2017). In total, 1,091,284 long reads were obtained with an $\mathrm{N}_{50}$ length of 8,169 for AlmaEM3, and 226,927 long reads reads with an $\mathrm{N}_{50}$ length of 7,320 were obtained for BB08-1. For AlmaEM3, 729,840 Illumina reads previously obtained for AlmaEM3 (NCBI BioProject PRJNA433735) were used (Potnis et al. 2019). For BB08-1, an Illumina library was prepared by and sequenced at the University of California-Davis Genome Center using the Novoseq platform, trimmed, and filtered with fastp v0.20.1, yielding 77,639,847 paired-end reads (Chen et al. 2018). Both sets of reads were evaluated with FastQC to verify quality and absence of sequencing adapters (Andrews 2010). For both strains, long reads were assembled with Flye v. 2.7.1 with parameters -nano-raw and -genome-size $2.5 \mathrm{M}$, the resulting single-molecule circular assembly rotated so that the start position corresponds to the first base of $d n a A$ (Kolmogorov et al. 2019), and subsequently polished with Illumina sequencing data using Pilon 1.23 (Walker et al. 2014). AlmaEM3 was polished four times, correcting 4,091 errors, whereas BB08-1 was polished five times with random subsamples of reads $(7,978,767$ unique reads) generated with Seqtk v1.3 (https://github. com/h3/seqtk), correcting 2,485 errors.

For both strains, average nucleic acid identity (ANI) to the complete genome of $X$. fastidiosa type strain ATCC35879 ${ }^{\top}$ was determined with fastANI v1.32 (Jain et al. 2018). CheckM v1.1.2 was used to assess assembly completeness, and IDEEL was used to estimate prevalence of erroneously frameshifted coding sequences (Parks et al. 2015; Stewart et al. 2019). STs were determined using the $X$. fastidiosa PubMLST database (Jolley et al. 2018) and were consistent with previous findings (i.e., ST 43 for BB08-1 and ST 42 for AlmaEM3) (Potnis et al. 2019). Assembly results and a comparison with existing draft assemblies are presented in Table 1. No plasmids were identified in either AlmaEM3 or BB08-1.

Genomic rearrangements within a bacterial strain can have both evolutionary and phenotypic implications, including gene duplication, loss, and altering gene expression; additionally, reversable rearrangements can facilitate phase variation (Cui et al. 2012; Periwal and Scaria 2015). Commonly, rearrangements are symmetrical translocation inversions between replichores that do not substantially alter the relative distance of genes contained within the translocated segment from the replication origin; asymmetrical rearrangements may have deleterious 


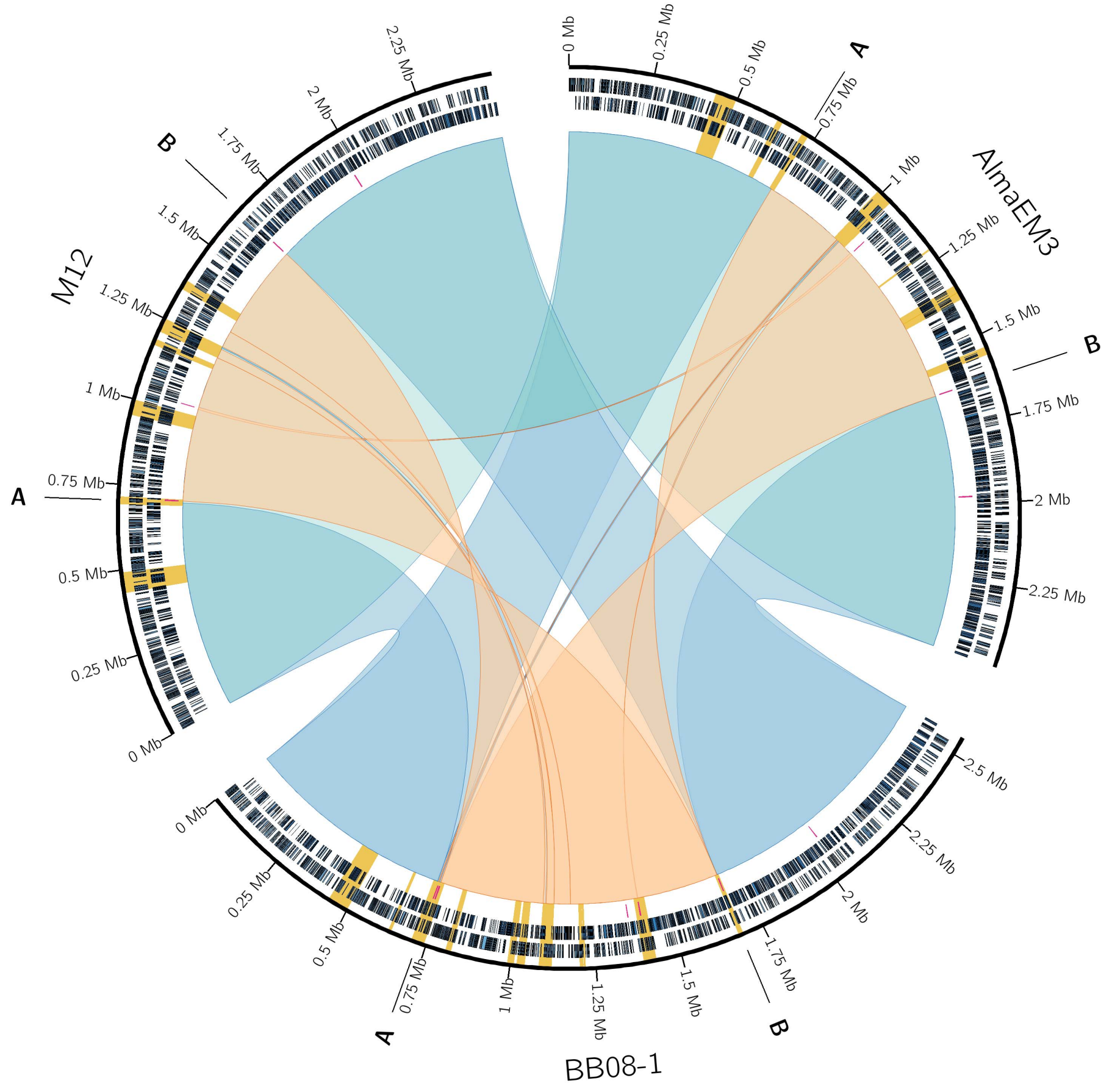

Fig. 1. Structural variation among blueberry-infecting Xylella fastidiosa subsp. multiplex strains. Circos visualization of progressiveMauve identified locally colinear blocks (LCBs). PHASTER-identified prophage regions in each genome are indicated by yellow wedges. Tracks one and two represent genes encoded on forward and reverse strands, respectively. Track three indicates nucmer-identified IS607-associated sequence in magenta. Ribbons represent LCBs identified by progressiveMauve pairwise comparisons between each genome. Parallel LCBs are colored blue, whereas inverted LCBs are orange. Flanking regions containing mobile elements or prophage sequence immediately upstream (A) or downstream (B) of the inversion in BB08-1, and corresponding regions in AlmaEM3 and M12, are indicated.

effects, particularly in fast-growing species (Couturier and Rocha 2006; Periwal and Scaria 2015). These rearrangements can be congruent with single-nucleotide polymorphism-based phylogenies (Darling et al. 2008).

Structural variation has been observed among $X$. fastidiosa strains both within and between subspecies, likely resulting from multiple rearrangement events; the biological relevance of such variation in $X$. fastidiosa is unclear (Chen et al. 2020; Van Sluys et al. 2003; Varani et al. 2013). These rearrangements are not necessarily symmetrical translocation inversions, and are likely the result of recombination between prophage regions. Analysis of AlmaEM3 and BB08-1 with PHASTER identified eight prophage regions in AlmaEM3, including 
three which are intact and one that corresponds to the $X$. fastidiosa phage Xfas53 and spans 47.6 kb. BB08-1 contains 11 prophage regions, 4 of which are intact, including 3 of 35.4 to $37.5 \mathrm{~kb}$ in length that correspond to Xfas53 (Arndt et al. 2016).

Pairwise whole-genome alignment with progressiveMauve v20150226, visualized with Circos, revealed that AlmaEM3 is colinear with M12 (Fig. 1), with the exception of a 7,836-bp inversion encompassing positions $1,063,923$ to $1,071,758$ and seven coding sequences (KBP49_RS04585 to KBP49_RS04615), including a glycosyl hydrolase and IS607-family transposase (Darling et al. 2010; Krzywinski et al. 2009). Two topoisomerase-primase domaincontaining proteins (RS04585 and RS04615), commonly involved in DNA replication and recombination, flank the region. These appear to be duplicate genes and form an inverted repeat. One of these genes (RS04585) is truncated relative to its positional homolog in M12 (XFASM12_RS04400); thus, it is plausible that this inversion occurred due to homologous recombination between RS04585 and RS04615.

BB08-1 contains a large inversion of approximately $0.95 \mathrm{Mb}$ relative to both AlmaEM3 and M12 (Fig. 1). This inversion is flanked by prophage regions, which are implicated in other chromosomal rearrangements observed in X. fastidiosa strains (Varani et al. 2013). In BB08-1 and $\mathrm{M} 12$, both upstream and downstream flanking regions (Fig. 1, regions $A$ and $B$, respectively) contain genes encoding an IS1607-family element and a transposase (KBP48_RS07590 to KBP48_RS07595, KBP48_RS03180 to KBP48_RS03185, and KBP48_RS03180 to KBP48_ RS03185). AlmaEM3 only contains these elements in the region downstream of the inversion (Fig. 1, region B; KBP49_RS07195 to KBP49_RS07200) (Fig. 1, region A). This suggests that the inversion event in BB08-1 resulted from recombination between these repetitive sequences.

Additional copies, both intact and partial, of the IS1607-family element and transposase in all three genomes were identified with nucmer v3.1 (Fig. 1) (Marçais et al. 2018). Additionally, some other small rearrangements involving these phage regions and the IS1607-family element are present, suggesting that multiple recombination events may have occurred. Although this inversion is large, it is relatively balanced between replichores and, thus, unlikely to be deleterious or substantially interfere with expression of genes located entirely within the inversion. This inversion is distinct from those observed in other strains of $X$. fastidiosa (e.g., strain 9a5c compared with Temecula-1). It is not known if this rearrangement is a common feature of ST 43 strains, blueberry-infecting strains with geographic origins in Florida, or a unique feature of BB08-1.

\section{Data Availability}

Genome sequences for AlmaEM3 and BB08-1 were deposited in GenBank and are associated with BioProject PRJNA720706. Sequencing reads used for genome assembly are available through Sequence Read Archive (SRA) accessions SRR15093612 for AlmaEM3 and SRR14917430 and SRR14917432 for BB08-1. Illumina reads used for AlmaEM3 were retrieved from SRA accession SRR6796157 (Potnis et al. 2019).

\section{Acknowledgments}

We thank B. Ortega and K. Zhang for technical assistance, as well as the Spanish Olive Oil Interprofessional Organization and the Thematic Interdisciplinary Platform on Xylella from CSIC (PTI-SolXyl) for its support.

\section{Literature Cited}

Andrews, S. 2010. FastQC: A quality control tool for high throughput sequence data. https://www.bioinformatics.babraham.ac.uk/projects/fastqc/

Arndt, D., Grant, J. R., Marcu, A., Sajed, T., Pon, A., Liang, Y. J., and Wishart, D. S. 2016. PHASTER: A better, faster version of the PHAST phage search tool. Nucleic Acids Res. 44:W16-W21.

Burbank, L., O'Leary, M., and Sisterson, M. 2019. Infection of blueberry cultivar Emerald with a California grapevine strain of Xylella fastidiosa and acquisition by glassy-winged sharpshooter. (Abstr.) Phytopathology 109:S2.143.

Chang, C. J., Donaldson, R., Brannen, P., Krewer, G., and Boland, R. 2009. Bacterial leaf scorch, a new blueberry disease caused by Xylella fastidiosa. HortScience 44:413-417.

Chen, J., O'Leary, M., Burbank, L., Zheng, Z., and Deng, X. 2020. Whole genome sequence of Xylella fastidiosa ATCC 35879(T) and detection of genome rearrangements within subsp. fastidiosa. Curr. Microbiol. 77:1858-1863.
Chen, S. F., Zhou, Y. Q., Chen, Y. R., and Gu, J. 2018. fastp: An ultra-fast all-inone FASTQ preprocessor. Bioinformatics 34:i884-i890.

Couturier, E., and Rocha, E. P. C. 2006. Replication-associated gene dosage effects shape the genomes of fast-growing bacteria but only for transcription and translation genes. Mol. Microbiol. 59:1506-1518.

Cui, L. Z., Neoh, H. M., Iwamoto, A., and Hiramatsu, K. 2012. Coordinated phenotype switching with large-scale chromosome flip-flop inversion observed in bacteria. Proc. Natl. Acad. Sci. U.S.A. 109:E1647-E1656.

Darling, A. E., Mau, B., and Perna, N. T. 2010. progressiveMauve: Multiple genome alignment with gene gain, loss and rearrangement. PLoS One 5:e11147.

Darling, A. E., Miklos, I., and Ragan, M. A. 2008. Dynamics of genome rearrangement in bacterial populations. PLOS Genet. 4:e1000128.

Di Genova, D., Lewis, K. J., and Oliver, J. E. 2020. Natural infection of Southern highbush blueberry (Vaccinium corymbosum interspecific hybrids) by Xylella fastidiosa subsp. fastidiosa. Plant Dis. 104:2598-2605. 
Ferguson, M. H., Clark, C. A., and Smith, B. J. 2017. Association of Xylella fastidiosa with yield loss and altered fruit quality in a naturally infected rabbiteye blueberry orchard. HortScience 52:1073-1079.

Harmon, P. F., and Hopkins, D. L. 2009. First report of bacterial leaf scorch caused by Xylella fastidiosa on Southern highbush blueberry in Florida. Plant Dis. 93:1220.

Jain, C., Rodriguez-R, L. M., Phillippy, A. M., Konstantinidis, K. T., and Aluru, S. 2018. High throughput ANI analysis of $90 \mathrm{~K}$ prokaryotic genomes reveals clear species boundaries. Nat. Commun. 9:5114.

Jolley, K. A., Bray, J. E., and Maiden, M. C. J. 2018. Open-access bacterial population genomics: BIGSdb software, the PubMLST.org website and their applications. Wellcome Open Res. 3:124.

Kolmogorov, M., Yuan, J., Lin, Y., and Pevzner, P. A. 2019. Assembly of long, errorprone reads using repeat graphs. Nat. Biotechnol. 37:540-546.

Krzywinski, M., Schein, J., Birol, I., Connors, J., Gascoyne, R., Horsman, D., Jones, S. J., and Marra, M. A. 2009. Circos: An information aesthetic for comparative genomics. Genome Res. 19:1639-1645.

Marçais, G., Delcher, A. L., Phillippy, A. M., Coston, R., Salzberg, S. L., and Zimin, A. 2018. MUMmer4: A fast and versatile genome alignment system. PLOS Comput. Biol. 14:e1005944.

Oliver, J. E., Cobine, P. A., and De La Fuente, L. 2015. Xylella fastidiosa isolates from both subsp. multiplex and fastidiosa cause disease on Southern highbush blueberry (Vaccinium sp.) under greenhouse conditions. Phytopathology 105:855-862.

Oliver, J. E., Sefick, S. A., Parker, J. K., Arnold, T., Cobine, P. A., and De La Fuente, L. 2014. lonome changes in Xylella fastidiosa-infected Nicotiana tabacum correlate with virulence and discriminate between subspecies of bacterial isolates. Mol. Plant-Microbe Interact. 27:1048-1058.

Parks, D. H., Imelfort, M., Skennerton, C. T., Hugenholtz, P., and Tyson, G. W. 2015. CheckM: Assessing the quality of microbial genomes recovered from isolates, single cells, and metagenomes. Genome Res. 25:1043-1055.

Periwal, V., and Scaria, V. 2015. Insights into structural variations and genome rearrangements in prokaryotic genomes. Bioinformatics 31:1-9.

Potnis, N., Kandel, P. P., Merfa, M. V., Retchless, A. C., Parker, J. K., Stenger, D. C., Almeida, R. P. P., Bergsma-Vlami, M., Westenberg, M., Cobine, P. A., and De La
Fuente, L. 2019. Patterns of inter- and intrasubspecific homologous recombination inform eco-evolutionary dynamics of Xylella fastidiosa. ISME J. 13:2319-2333.

Stewart, R. D., Auffret, M. D., Warr, A., Walker, A. W., Roehe, R., and Watson, M. 2019. Compendium of 4,941 rumen metagenome-assembled genomes for rumen microbiome biology and enzyme discovery. Nat. Biotechnol. 37:953961.

Vanhove, M., Retchless, A. C., Sicard, A., Rieux, A., Coletta-Filho, H. D., De La Fuente, L., Stenger, D. C., and Almeida, R. P. P. 2019. Genomic diversity and recombination among Xylella fastidiosa subspecies. Appl. Environ. Microbiol. 85: e02972-18.

Van Sluys, M. A., de Oliveira, M. C., Monteiro-Vitorello, C. B., Miyaki, C. Y., Furlan, L. R., Camargo, L. E., da Silva, A. C., Moon, D. H., Takita, M. A., Lemos, E. G., Machado, M. A., Ferro, M. I., da Silva, F. R., Goldman, M. H., Goldman, G. H., Lemos, M. V., El-Dorry, H., Tsai, S. M., Carrer, H., Carraro, D. M., de Oliveira, R. C., Nunes, L. R., Siqueira, W. J., Coutinho, L. L., Kimura, E. T., Ferro, E. S., Harakava, R., Kuramae, E. E., Marino, C. L., Giglioti, E., Abreu, I. L., Alves, L. M., do Amaral, A. M., Baia, G. S., Blanco, S. R., Brito, M. S., Cannavan, F. S., Celestino, A. V., da Cunha, A. F., Fenille, R. C., Ferro, J. A., Formighieri, E. F., Kishi, L. T., Leoni, S. G., Oliveira, A. R., Rosa, V. E., Jr., Sassaki, F. T., Sena, J. A., de Souza, A. A., Truffi, D., Tsukumo, F., Yanai, G. M., Zaros, L. G., Civerolo, E. L., Simpson, A. J., Almeida, N. F., Jr., Setubal, J. C., and Kitajima, J. P. 2003. Comparative analyses of the complete genome sequences of Pierce's disease and citrus variegated chlorosis strains of Xylella fastidiosa. J. Bacteriol. 185:1018-1026.

Varani, A. M., Monteiro-Vitorello, C. B., Nakaya, H. I., and Van Sluys, M. A. 2013. The role of prophage in plant-pathogenic bacteria. Annu. Rev. Phytopathol. 51: 429-451.

Walker, B. J., Abeel, T., Shea, T., Priest, M., Abouelliel, A., Sakthikumar, S., Cuomo, C. A., Zeng, Q. D., Wortman, J., Young, S. K., and Earl, A. M. 2014. Pilon: An integrated tool for comprehensive microbial variant detection and genome assembly improvement. PLoS One 9:e112963.

Wick, R. R., Judd, L. M., Gorrie, C. L., and Holt, K. E. 2017. Completing bacterial genome assemblies with multiplex MinION sequencing. Microb. Genomics 3. 